\title{
Segment Self-Guide Reconstruction Algorithm Based on Object-Oriented Quantization
}

\author{
Xuanqin Mou, Hengyong Yu, and Yuanlong Cai \\ Institute of Image Processing, School of Electronics and Information Engineering \\ Xi'an Jiaotong Univ., Xi'an, Shaanxi, P.R.C, 710049 \\ \{xqmou, ylcai\}@xjtu.edu.cn. yuhengyong@hotmail.com
}

\begin{abstract}
Aiming at the problem of inaccurate imaging model of threedimensional (3D) reconstruction of rotational DSA (digital subtraction angiography) images, firstly a nonlinear model based on object-oriented quantization is introduced. The model quantizes the projective pixel of 3D vessel slice as the vessel number that the X-ray goes through. Then, under the constraint of limited views and sparse projections, a slice reconstruction algorithm named segment self-guide reconstruction (SSGR) is developed. It converts the slice reconstruction of $\mathrm{N}+1$ level nonlinear quantized DSA image to the reconstruction of $\mathrm{N}$ vessel cross-sections. The SSGR is especially suitable for solving the problem of sparse projections and limited-views. Finally, the simulated results have proved the feasibility of the model and the validity of the algorithm.
\end{abstract}

\section{Introduction}

The 3D reconstruction of the vessel has been widely studied all over the world in recent years, since it is significant in the diagnosis and therapy of all kinds of vessel diseases. The traditional reconstructive methods can be mainly divided into two parts: axial and cross-sectional reconstruction. As to the vessel cross-sectional reconstruction, since the generation of digital X-Ray images is a complex process that includes production, conversion and transfer of the X-Ray information, the gray level of X-Ray images can't show its exact physical meanings, and the imaging model doesn't abide by Lamber-Beer's Law. Therefore the traditional methods are mainly to search the optimal parameters of the prior elliptical model [1-2].

In the reconstruction of rotational DSA, there are many available projective data of different angles, and the edges of vessels can be precisely detected, which is independent of imaging model. Thus a new vessel projective and reconstructive model is presented, which does not need the reconstruction of axis. This model is a binary 3D vessel whose voxel will be ' 1 ' if it belongs to vessel volume otherwise it will be ' 0 '. It

1 This research was supported by the foundation for university key teacher by the ministry of education and national natural science foundation (no.30070225) of china. 
also can be considered as a stack piled up by a series of slices, and each slice is a 2D binary image. A DSA image is a projection of the 3D vessel model in certain angle and the projection of a slice is a line in the DSA image.

To reconstruct 3D vessel, we have developed a nonlinear quantitative model [3], where a pixel of DSA image can be quantized as the number of vessels that the X-Ray has gone through. In reference [3], it is pointed out that binary slice image can be reconstructed by optimal square-wave decomposition (OSWD) algorithm under the condition of more fully views projections (at least 100). OSWD has exact physical meaning, but it doesn't suit to real computation because of its rigorous requirement. As to sparse projections and limited views, the OSWD can't get the approving reconstruction result. To overcome the shortcoming of OSWD, another reconstructive algorithm named segment self-guide reconstruction (SSGR) is developed. The core of SSGR is the slice reconstruction can be converted to the reconstruction of $\mathrm{N}$ vessel cross-sections.

\section{Object-Oriented Quantization Model}

Based on an assumption of parallel ray geometry, a binary projection model of the slice is introduced [4-5], which can be described by Boolean Radon Transform (BRT). Under Boolean OR Integral operator, a projective pixel of certain angle about the binary image can be quantized as ' 0 ' or ' 1 '. If there is at least one pixel which belongs to vessel cross-section on the direction of the projective pixel, it will be quantized as ' 1 ', otherwise, it will be ' 0 '. All projections of a point at different angles can form a sinusoid in the projective image. Based on the BRT, a nonlinear quantitative model of DSA image is presented. A pixel of DSA image can be quantized as $0,1, \cdots N$, which represents the number of vessels that the X-Ray has gone through. It is called object-oriented quantization in the paper.

\subsection{Definition of Boolean OR Integral \& Boolean AND Integral}

As a subset of bounded closed regions $\mathbf{D}, \mathbf{S}$ satisfies $\mathbf{S} \subseteq \mathbf{D}$, whose indicator function can be defined as the following.

$$
f_{\mathbf{s}}(\mathbf{X})= \begin{cases}1 & \mathbf{X} \in \mathbf{S} \\ 0 & \text { other }\end{cases}
$$

The operator $\cup \int_{\mathbf{Z}}$ on $\mathbf{z} \subset \mathbf{D}$ is called Boolean OR Integral, if it satisfies equation (2).

$$
\cup \int_{\mathbf{Z}} f_{\mathbf{s}}(\mathbf{X})=\left\{\begin{array}{cc}
1 & \exists \mathbf{X} \in \mathbf{z}, f_{\mathbf{s}}(\mathbf{X})=1 \\
0 & \text { other }
\end{array}\right.
$$

The operator $\cap \int_{\mathbf{z}}$ on $\mathbf{z} \subset \mathbf{D}$ is called Boolean AND Integral, if it satisfies equation 


$$
\cap \int_{\mathbf{Z}} f_{\mathbf{s}}(\mathbf{X})=\left\{\begin{array}{cc}
1 & \forall \mathbf{X} \in \mathbf{z}, f_{\mathbf{s}}(\mathbf{X})=1 \\
0 & \text { other }
\end{array}\right.
$$

In the later discussion, $f_{\mathbf{s}}(\mathbf{X})$ will be written as $f(\mathbf{X})$ if there are no confusing factors.

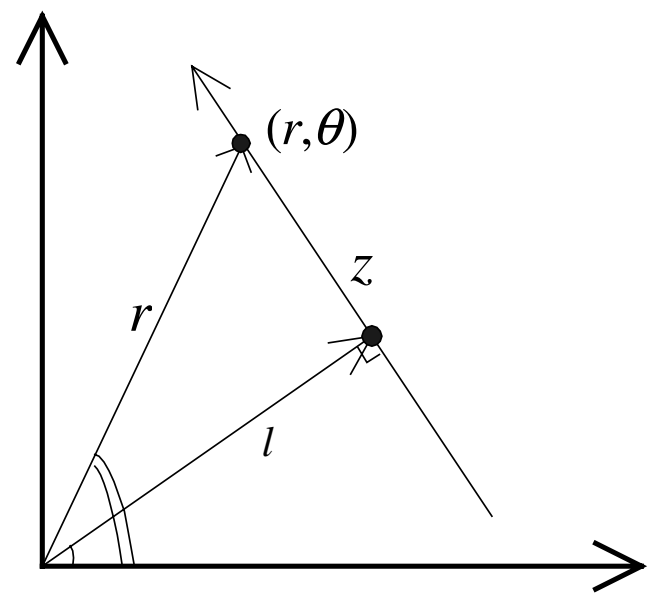

Fig. 1. Coordinate system of BRT

\subsection{Definition of BRT \& Inverse BRT}

Shown as the coordinate system in figure 1, the Boolean OR Integral of a certain set's indicator function $f(x, y)=\hat{f}(r, \theta)$ along the line $z$ is called Boolean Radon Transform.

$$
p(\phi, l)=\cup \int_{-\infty}^{\infty} f(x, y) d z=\cup \int_{-\infty}^{\infty} \hat{f}\left(\sqrt{l^{2}+z^{2}}, \phi+\operatorname{tg}^{-1} \frac{z}{l}\right) d z
$$

If one takes the acquisition process of the rotational DSA into account, the definition domain of Boolean Radon Transform is assumed to be a closed domain as $\mathbf{D}=\left\{(x, y) \mid x^{2}+y^{2} \leq R^{2}\right\}$. Since there are two directions of a straight line, the value region of BRT can be defined as a rectangle $\mathbf{B}=\{(\phi, l)|0 \leq \phi<\pi| l \mid, \leq R\}$. Thus, the inverse BRT can be defined as:

$$
f(x, y)=\hat{f}(r, \theta)=\cap \int_{0}^{\pi} p(\phi, r \cos (\phi-\theta)) d \phi
$$




\subsection{Object-Oriented Quantization Model}

Denoted $p_{i}(\phi, l)(i=1,2, \cdots N)$ as a BRT of certain vessel cross-section, whose values can be ' 0 ' or ' 1 '. The $\mathrm{N}+1$ level vessel quantization model, which can distinguish $\mathrm{N}$ vessels at best, can be descried as [3]:

$$
P(\phi, l)=\sum_{i=1}^{N} p_{i}(\phi, l)
$$

Where the value of $P(\phi, l)$ can be $0,1,2, \cdots N$ and $\sum$ represents the plain add operation. Fig.2 (a) is a slice image of simulated 3D vessel and (b) is the corresponding nonlinear quantized result.

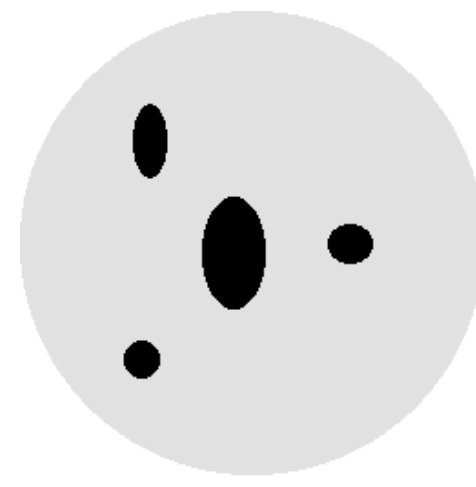

(a) 3D vessel slice image

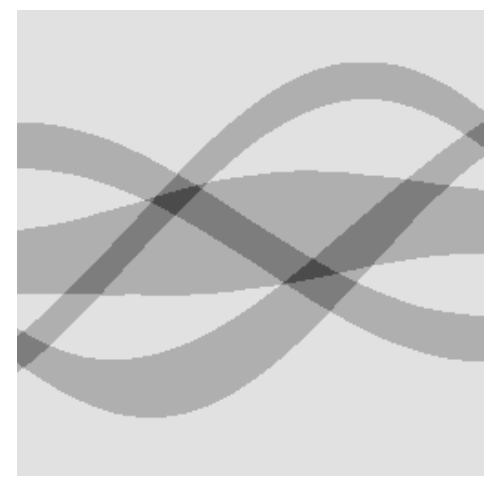

(b) Nonlinear quantized result

Fig. 2. 3D vessel slice image and its quantized result

\section{Segment Self-Guide Reconstruction Algorithm}

We have developed OSWD algorithm to reconstruct the binary slice of 3D vessel [3]. However there are some noises in the reconstructed slice. The reason is that OSWD is a model in continuous domain, but the processed data are discrete and the number of projections is limited. The noises can be divided into 2 classes: one is caused by the positional quantized error that are all located at the edge of projective region; the other is caused by the small square wave width that is smaller than the unitary discrete length. Simulated results show that the number of projections must be increased to improve the reconstructive precision. In fact, one can at most get about 100 projections by rotational DSA system one time. To overcome this shortcoming, we design another reconstructive method, that is SSGR (segment self-guide reconstruction) algorithm.

It is known that the BRT $p_{i}(\phi, l)$ of single vessel cross-section is a stripe-like subset in set $\mathbf{B}$ [4]. The subset can be described by its upside bound and downside bound, 
that is, it can be determined by its bound support function (BSF). The quantized result of a slice projection is the overlapped result of several stripe-like subsets, whose upside bound can be defined as $\left\{P\left(\phi, l_{i}\right) \mid P\left(\phi, l_{i}\right)>P\left(\phi, l_{i+1}\right)\right\}$ and downside bound can be defined as $\left\{P\left(\phi, l_{i}\right) \mid P\left(\phi, l_{i}\right)>P\left(\phi, l_{i-1}\right)\right\}$, where $i$ represents the index of discrete image pixel.

Under the constraint of limited views and sparse projections, the slice reconstruction of $N+1$ level nonlinear quantized DSA image can be converted to the reconstruction of $N$ vessel cross-sections. Therefore, SSGR algorithm can be described as the following steps.

Step1: The number of vessel cross-sections $\mathrm{N}$ is counted using the method mentioned in [3]. We reconstruct the CRSI (coarse reconstructed slice image) using inverse BRT [4], where $P(\phi, l)$ is regarded as ' 1 ' if it unequals to ' 0 '.

Step2: CRSI is segmented and marked as independent regions. $\mathrm{N}$ regions, that every region's area is larger than all the others, are selected as the candidate vessel crosssections.

Step3: Applied BRT to every candidate vessel cross-section, one can get the candidate BRT image $\tilde{p}_{i}(\phi, l)$. Denoting the BRT image of the corresponding real vessel crosssection as $p_{i}(\phi, l)$, there is $p_{i}(\phi, l) \subseteq \tilde{p}_{i}(\phi, l)$. Thus $p_{i}(\phi, l)$ can be estimated from $\tilde{p}_{i}(\phi, l)$, where $\tilde{p}_{i}(\phi, l)$ acts as a guide model.

Step4: The candidate vessel cross-sections are sorted by area from large to small. Beginning with the maximum area, find the nearest upside and downside bound in $\tilde{p}_{i}(\phi, l)$, and one can get the estimation $\hat{p}_{i}(\phi, l)$ of $p_{i}(\phi, l)$. Then one deletes $\hat{p}_{i}(\phi, l)$ from $P(\phi, l)$. Using the bound support function extracted from $\hat{p}_{i}(\phi, l)$, one can reconstruct the single vessel cross-section [4 5]. Under the condition of sparse projections, the estimation of $p_{i}(\phi, l)$ is very complex. Because many information are undeterminable, $\hat{p}_{i}(\phi, l)$ is not unique and the reconstructed image is not unique either. We estimated $\hat{p}_{i}(\phi, l)$ with the maximum prior probability (see appendix).

Step5: Reconstruct the final binary slice image by combining all the image of single vessel cross-section.

It must be pointed out that there are two presuppositions for SSGR algorithm. 1) For every vessel cross-section, there at least exists one projection in which the crosssection does not overlap with any other cross-sections. Thus all candidate vessel crosssections can be segmented from each other. 2) The segmented regions caused by noise are smaller than all that caused by vessel cross-sections. Therefore the largest $\mathrm{N}$ regions are caused by real vessel cross-sections.

\section{Simulated Results and Discussions}

Aiming at validating the feasibility of SSGR under the constraint of sparse projections, the simulated binary slice in Fig.2 is reconstructed with 4 and 8 uniformly distribution projections. The percent errors are $11.97 \%$ and $7.07 \%$ respectively, where the percent error is defined as the number of total error pixels dividing the number of total pixels. Obviously, the larger the number of projections is, the higher the reconstruction preci- 
sion is. Under the constraint of limited views, one can get the same conclusion and it will not be discussed in detail. Fig. 3 and Fig. 4 are the reconstructed results of 4 and 8 projections respectively.
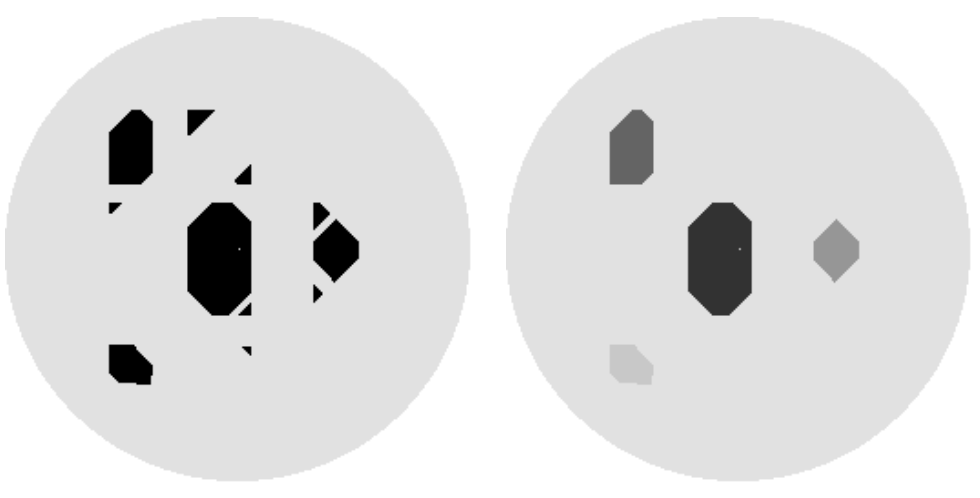

(a) CRSI

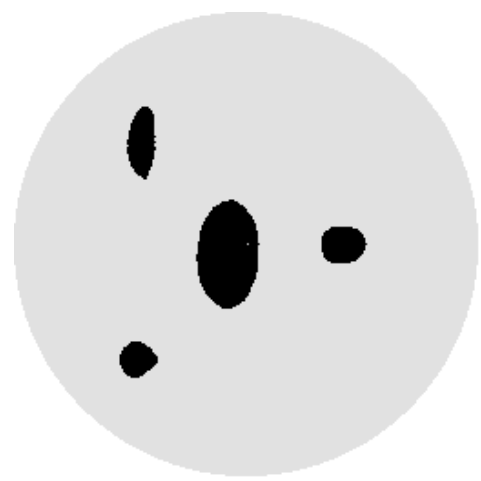

(c) Reconstructed result (b) Candidate vessel cross-sections

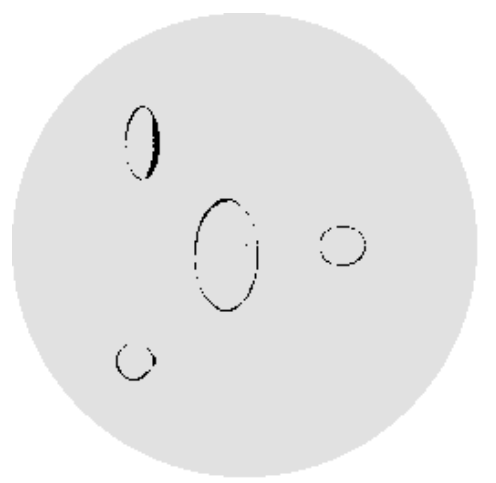

(d) Reconstructed error

Fig. 3. SSGR reconstructed result of 4 projections

\section{Conclusion}

Based on the nonlinear quantization model, SSGR algorithm is developed to reconstruct binary slice. The algorithm is especially suitable for the problems of sparse projections and limited views. SSGR can overcome the shortcomings of OSWD though its implementation is more complex than OSWD's. However, SSGR is suitable for the slices in which vessel cross-sections are convex. Combining the gray level of DSA image, we expect to solve the problem of concave cross-sections in the future research. 

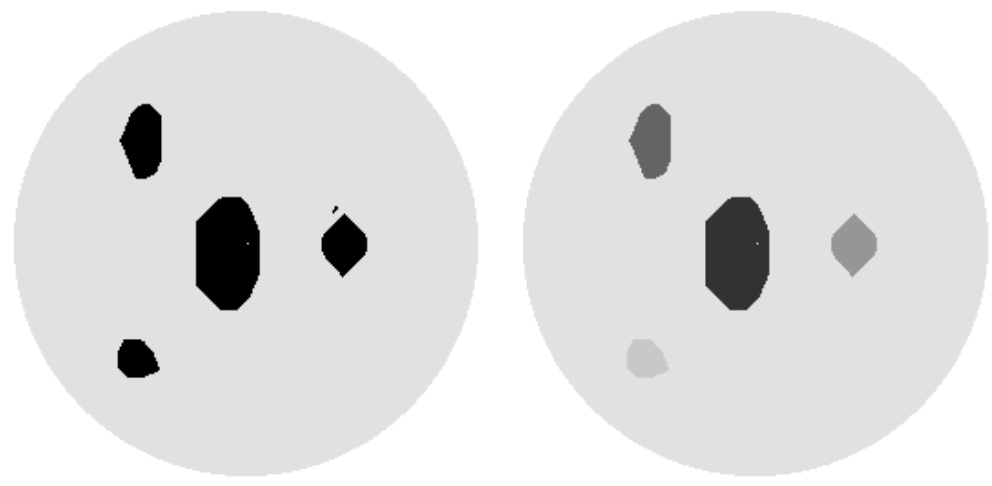

(a) CRSI

(b) Candidate vessel cross-sections

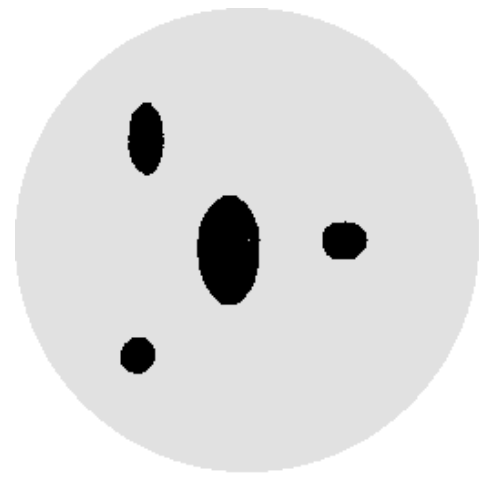

(c) Reconstructed result

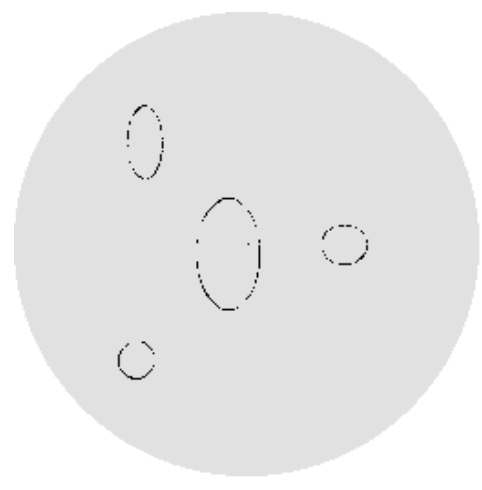

(d) Reconstructed error

Fig. 4. SSGR reconstructed result of 8 projections

\section{References}

1. G P M Prause, D G W. Onnasch Binary Reconstruction of the Heart Chambers from Biplane Angiographic Image Sequences. IEEE Transactions on Medical Imaging, 1996, 15(4):532-546.

2. Pellot C, Herment A, Sigelle M, et al. A 3D Reconstruction of Vascular Structures from Two X-Ray Angiograms Using an Adapted Simulated Annealing Algorithm. IEEE Transactions on Medical Imaging, 1994,13(1):48-60.

3. Yu Hengyong, Mou Xuanqin, Wang Yong, et al. 3D Reconstruction Model of Vessel Based on Object-Oriented Quantization, in Medical Image Acquisition and Processing, Jayaram K. Udupa, Aaron Fenster, Editors, Proceedings of SPIE Vol. 4549, 2001:75-79.

4. Yu hengyong, Mou Xuanqin, Wang Yong, and Cai Yuanlong, "Properties of Boolean Radon Transform", Journal of Xi' an Jiaotong University, 2001, 35(10):1012-1016 .

5. Yu hengyong, Mou Xuanqin, Wang Yong, and Cai Yuanlong, "Further research on the properties of Boolean Radon Transform and its application", Journal of Xi'an Jiaotong University, 2001,35(12):1236-1239. 


\section{Appendix: The Uncertainty of Reconstruction under Sparse Projections}

Shown as in Fig.5, assume $\phi_{0}$ is constant and all the values of $\tilde{p}_{i}\left(\phi_{0}, l\right)$ are ' 1 ', where $l \in\left[l_{d}, l_{u}\right]$. Then the upside bound and downside bound of $p_{i}\left(\phi_{0}, l\right)$ are in the interval $\left[l_{d}, l_{u}\right]$. One can use the nonlinear quantization of $P(\phi, l)$ in $\left[l_{d}, l_{u}\right]$ to estimate the bounds of $p_{i}\left(\phi_{0}, l\right)$. The bounds of $p_{i}\left(\phi_{0}, l\right)$ may have many possible patterns because of the complex of overlaps. Denote the upside bound as $U$ and the downside bound as $\mathrm{D}$, the number of total bounds will be no less than 2 . For 2,3 and 4 bounds, the candidate patterns of $p_{i}\left(\phi_{0}, l\right)$ are listed in table 1 . One candidate bound pattern is composed of an upside and a downside bound.

From table 1, one can get a conclusion that the number of patterns increases rapidly with the increase of total bounds. Noting that the pattern $U_{1} D_{1}$ have the maximum possibility as the bounds of $p_{i}\left(\phi_{0}, l\right)$, we select $\mathrm{U}_{1} \mathrm{D}_{1}$ as the estimated bounds of $p_{i}\left(\phi_{0}, l\right)$. Therefore, $\mathrm{U}_{1}$ and $\mathrm{D}_{1}$ are called nearest upside bound and nearest downside bound respectively. The above is the principle of SSGR in step 4.

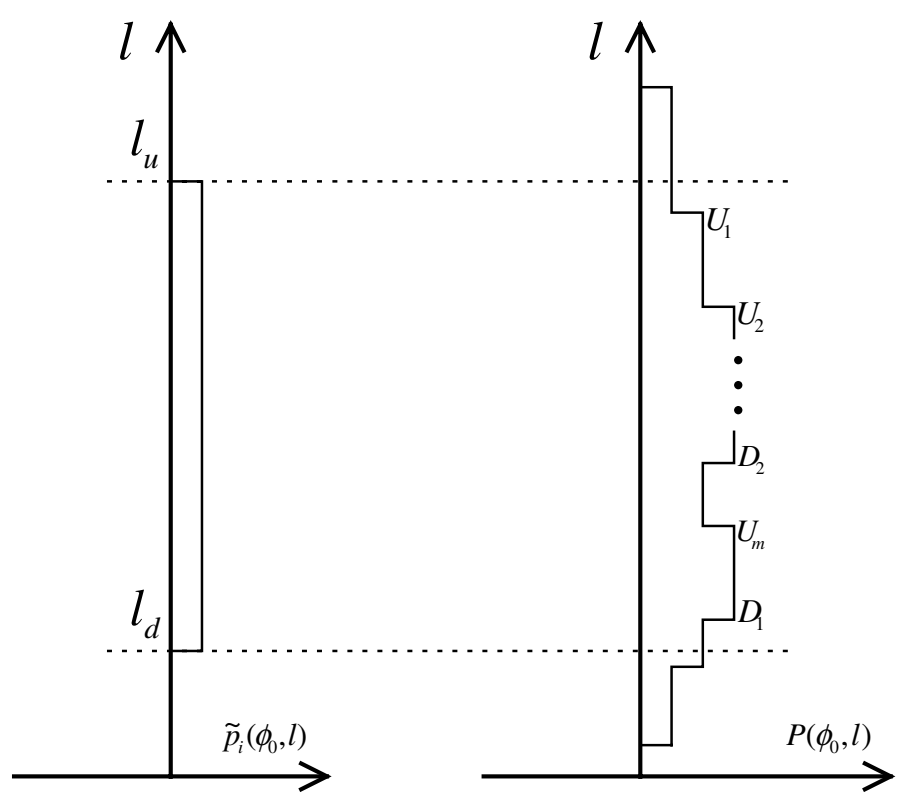

Fig. 5. The guided search process 
Table 1. Possible bounds of $p_{i}\left(\phi_{0}, l\right)$

\begin{tabular}{|l|l|l|}
\hline $\begin{array}{c}\text { Total } \\
\text { bounds }\end{array}$ & Distributions of bounds & Candidate bound patterns of $p_{i}\left(\phi_{0}, l\right)$ \\
\hline 2 & $\mathrm{D}_{1} \mathrm{U}_{1}$ & $\mathrm{D}_{1} \mathrm{U}_{1}$ \\
\hline \multirow{5}{*}{3} & $\mathrm{D}_{1} \mathrm{D}_{2} \mathrm{U}_{1}$ & $\mathrm{D}_{1} \mathrm{U}_{1}, \mathrm{D}_{2} \mathrm{U}_{1}$ \\
\cline { 2 - 3 } & $\mathrm{D}_{1} \mathrm{U}_{1} \mathrm{D}_{2}$ & $\mathrm{D}_{1} \mathrm{U}_{1}$ \\
\cline { 2 - 3 } & $\mathrm{D}_{1} \mathrm{U}_{2} \mathrm{U}_{1}$ & $\mathrm{D}_{1} \mathrm{U}_{1}, \mathrm{D}_{1} \mathrm{U}_{2}$ \\
\cline { 2 - 3 } & $\mathrm{U}_{2} \mathrm{D}_{1} \mathrm{U}_{1}$ & $\mathrm{D}_{1} \mathrm{U}_{1}$ \\
\hline \multirow{5}{*}{} & $\mathrm{D}_{1} \mathrm{U}_{3} \mathrm{U}_{2} \mathrm{U}_{1}$ & $\mathrm{D}_{1} \mathrm{U}_{1}, \mathrm{D}_{2} \mathrm{U}_{2}, \mathrm{D}_{3} \mathrm{U}_{3}$ \\
\cline { 2 - 3 } & $\mathrm{U}_{3} \mathrm{D}_{1} \mathrm{U}_{2} \mathrm{U}_{1}$ & $\mathrm{D}_{1} \mathrm{U}_{1}, \mathrm{D}_{1} \mathrm{U}_{2}$ \\
\cline { 2 - 3 } & $\mathrm{U}_{3} \mathrm{U}_{2} \mathrm{D}_{1} \mathrm{U}_{1}$ & $\mathrm{D}_{1} \mathrm{U}_{1}$ \\
\cline { 2 - 3 } & $\mathrm{D}_{1} \mathrm{D}_{2} \mathrm{U}_{2} \mathrm{U}_{1}$ & $\mathrm{D}_{1} \mathrm{U}_{1}, \mathrm{D}_{1} \mathrm{U}_{2}, \mathrm{D}_{2} \mathrm{U}_{1}, \mathrm{D}_{2} \mathrm{U}_{2}$ \\
\cline { 2 - 3 } & $\mathrm{D}_{1} \mathrm{U}_{2} \mathrm{D}_{2} \mathrm{U}_{1}$ & $\mathrm{D}_{1} \mathrm{U}_{1}, \mathrm{D}_{2} \mathrm{U}_{1}$ \\
\cline { 2 - 3 } & $\mathrm{U}_{2} \mathrm{D}_{1} \mathrm{D}_{2} \mathrm{U}_{1}$ & $\mathrm{D}_{1} \mathrm{U}_{1}, \mathrm{D}_{2} \mathrm{U}_{1}$ \\
\cline { 2 - 3 } & $\mathrm{D}_{1} \mathrm{U}_{2} \mathrm{U}_{1} \mathrm{D}_{2}$ & $\mathrm{D}_{1} \mathrm{U}_{1}, \mathrm{D}_{1} \mathrm{U}_{2}$ \\
\cline { 2 - 3 } & $\mathrm{U}_{2} \mathrm{D}_{1} \mathrm{U}_{1} \mathrm{D}_{2}$ & $\mathrm{D}_{1} \mathrm{U}_{1}$ \\
\cline { 2 - 3 } & $\mathrm{D}_{1} \mathrm{D}_{2} \mathrm{D}_{3} \mathrm{U}_{1}$ & $\mathrm{D}_{1} \mathrm{U}_{1}, \mathrm{D}_{2} \mathrm{U}_{1}, \mathrm{D}_{3} \mathrm{U}_{1}$ \\
\cline { 2 - 3 } & $\mathrm{D}_{1} \mathrm{D}_{2} \mathrm{U}_{1} \mathrm{D}_{3}$ & $\mathrm{D}_{1} \mathrm{U}_{1}, \mathrm{D}_{2} \mathrm{U}_{1}$ \\
\cline { 2 - 3 } & $\mathrm{D}_{1} \mathrm{U}_{1} \mathrm{D}_{2} \mathrm{D}_{3}$ & $\mathrm{D}_{1} \mathrm{U}_{1}$ \\
\hline
\end{tabular}

